and $92 \%$ in triplets. The overall incidence of infants with low birth weight $(<2,500 \mathrm{gm})$ was $32 \%, 14 \%$ in singletons, $53 \%$ in twins and $92 \%$ in triplets. The overall incidence of very low birth weight infants $(<1,500 \mathrm{gm})$ was $6 \%, 4 \%$, in singletons, $8 \%$ in twins and $22 \%$ in triplets.

We conclude that IVF babies have a higher stillbirth and perinatal death rates. The multiple pregnancy seems to be the major risk factor determining the perinatal outcome. With the availability of embryo cryopreservation replacing 2 to 3 embryos per cycle might provide the answer to improving the perinatal outcome even if this meant a drop or a decrease in the pregnancy rate. IVF programmes have to achieve a delicate balance between achieving a high success rate and at the same time optimising the perinatal outcome.

\section{Session 22. Assisted procreation}

122. A randomized study of two culture media for IVF

A. Sunde, V. von Düring, B. Lippe, J. Siegel, J. Kahn, and K. Molne

Department of Obstetrics and Gynaecology, University Hospital of Trondheim, Norway

We have performed a randomized study of two different culture media for IVF (Earle's-BSS, Sigma Inc. U.S.A.) supplemented with penicillin, Na-pyruvate and heat inactivated patient serum and a defined medium: "GEA-medium" (EBSS-P) containing human serum albumin $(10 \mathrm{mg} / \mathrm{ml})$ and penicillin-streptomycin, used as supplied by GEA ltd. BioTech, Denmark.

The patients were randomized to have all their gametes and embryos cultured in one of the two culture media (205 and 206 oocyte recoveries respectively). The same number of oocytes were recovered (9.4) in two patient groups, the same number of embryos were replaced (2.6) and the same number of spare embryos were cryopreserved (1.1). The fertilization rate (75\%) and the oocyte and embryo quality (as judged by a scoring system) were similar in the two patient groups.

The clinical pregnancy rate was higher in the GEA-group $(28.6 \%, 53 / 185)$ compared to the Earles group $(21.9 \%, 40 / 182)$. The implantation rate was significantly higher in the GEA-group (65 implants, 13.5\%), than in the Earles group (46 implants, 9.3\%). The pregnancy loss was somewhat higher in the GEA group and the ongoing pregnancy rate per embryo replacement in the two groups was therefore not statistically different (GEA group: $11.8 \%$, Earles group: $10.9 \%$ ).

In IVF, the use of the GEA medium for the culturing of human gametes and embryos gives at least as good results as the use of Earles BSS supplemented with heat inactivated patient serum. The use of the GEA medium is more convenient and avoids the problems associated with the handling and use of human serum. Since the beginning of March this year, we have only used the GEA medium for the culture of human gametes and embryos in IVF.
123. Treatment outcome potential of pre-embryos

\section{depends on implantation}

B.J. Roozenburg, G.J. Huisman, G.H. Zeilmaker, A. Alberda, R.A. Leerentveld

Department of Obstetrics and Gynaecology, Academic Hospital Dijkzigt, Rotterdam, The Netherlands

In the IVF programme of the Academic Hospital Dijkzigt Rotterdam in 1989, 669 ovum pick-ups and 539 embryo transfers were performed, resulting in 144 clinical pregnancies. When 1,2 or 3 embryos developed after fertilization in vitro, all were transferred. However, in the presence of supernumerary embryos, selection of the best embryos on basis of morphologic features could be made. If the embryos were scored to be of excellent quality, the number of transferred embryos was limited to 3. When the embryos were considered to be of lower quality, one extra embryo was transferred in order to enhance the pregnancy prospects of the couple. The implantation rate per transferred embryo, the pregnancy rate per transfer and the multiple pregnancy rate per ongoing pregnancy were calculated for 3 group of patients.

Group A: patients having only 3 embryos;

Group B: patinets who had their transfer limited to 3 embryos because of excellent quality;

Group C: patients with lower quality embryos who had one extra embryo transferred.

$\begin{array}{llll} & \text { A } & \text { B } & \text { C } \\ n^{\circ} \text { viable gestational sacs } & 16 / 231 & 42 / 243 & 77 / 940 \\ n^{\circ} \text { of embryos transferred (\%) } & (7 \%) & (17 \%) & (8 \%) \\ & & & \\ \text { n'ongoing pregnancies } & 11 / 77 & 23 / 81 & 54 / 235 \\ n^{\circ} \text { of transfers (\%) } & (14 \%) & (28 \%) & (23 \%) \\ & & & \\ n^{\circ} \text { ongoing multiple pregn. } & 5 / 11 & 16 / 23 & 15 / 54 \\ n^{\circ} \text { of ongoing pregnancies (\%) } & (45 \%) & (70 \%) & (28 \%) \\ & & & \\ n^{\circ} \text { viable pregnancies } & 11 / 14 & 23 / 29 & 54 / 76 \\ n^{\circ} \text { of clinical pregnancies (\%) } & (79 \%) & (79 \%) & (71 \%)\end{array}$

Conclusions. The implantation rate of good quality embryos was significantly higher than of lower qquality embryos. Transfer of 3 good quality embryos results in a higher ongoing pregnancy rate than transfer of 3 or 4 lower quality embryos. The treatment outcome of patients with poor quality embryos can be improved by transferring one extra embryo. The embryo quality did not have an influence on the abortion rate. Due to the lower implantation rate of poor quality embrryos, multiple gestation did not occur more frequently in patients receiving 4 embryos.

124. Assessment of ultrarapid and slow freezing procedures fro mouse one-cell and four-cell embryos

J. Liu, E. Van den Abbeel, and A. Van Steirteghem

Centre for Reproductive Medicine, Academisch Ziekenhuis, Vrije Universiteit Brussel, Brussels, Belgium

Three cryopreservation procedures were assessed for the freezing of mouse one-cell and four-cell embryos, obtained from superovulated F1 hybrids (C57Bl x CBA/Ca). The slow freezing protocols with dimethylsulfoxide-DMSO (A) and propanediolPROH (B) and the ultrarapid procedure with DMSO (C), which 
was described by the Monash University group (Fertil Steril $48: 843-850,1987)$. The evaluation of the different procedures included the survival after freezing and thawing, the further development after culture in vitro to blastocysts $\left(37^{\circ} \mathrm{C}, 5 \% \mathrm{CO}_{2}\right.$ in air, M16 medium) and the ability to implant and to form living fetuses after transfer of blastocysts to pseudopregnant mice. The experimental embryos were compared to appropriate controls which were not frozen and thawed. The survival and further development in vitro was lower in all frozen embryos than in the controls. Four-cell embryos scored better in protocols A and B than zygotes. Procedures A and B induced comparable results and were signifcantly better than the ultrarapid freezing procedure $C$. When unfrozen blastocysts were transferred to pseudopregnant mice $64 \%$ of them implanted in the uterine wall and 59\% developed to living fetuses. Both parameters were lower when embryos were frozen and thawed. For zygotes the percentage of implantation sites and living fetuses were $47 \%$ and $33 \%$ for A, $50 \%$ and $44 \%$ for B and $29 \%$ and $17 \%$ for C. When four-cell embryos were cryopreserved, these results were $48 \%$ and $45 \%$ for $A, 60 \%$ and $53 \%$ for $B$ and $37 \%$ and $23 \%$ for $C$. In the ultrarapid procedure we also controlled the influence of the freezing solution. Mouse zygotes and four-cell embryos were exposed to DMSO as in the ultrarapid freezing procedure except that they were not frozen. Compared to controls, the survival and blastocysts formation was not different, but less living fetuses were born ( $25 \%$ for zygotes and $41 \%$ for 4 -cell embryos versus $66 \%$ in the controls).

This study demonstrated that mouse zygotes can be frozen less efficiently than four-cell embryos. Slow freezing protocols yielded better results than the ultrarapid method with $3.5 \mathrm{M}$. DMSO. This phenomenon can be partly explained by the cryoprotectant solution.

\section{Pregnancies from embryos without evidence of fertilization}

P.M. Rijnders, E.Y. Jaarsma, H.C. van Os, M. TombrockScheffer and C.A.M. Jansen

Department of Obstetrics,Gynaecology, and IVF, Diaconessenhuis Voorburg, The Netherlands

In 1989490 IVF cycles were started in our centre of which 27 cycles were cancelled and 2 were excluded from this research. Embryo Transfer (ET) took place in 355 cases, $78 \%$ of IVF procedures. In total 101 clinical pregnancies occurred from IVF cycles, (excluding 2 pregnancies from transfer of cryopreserved embryos and 1 from oocyte donation). In 456 cycles one or more oocytes were recovered; a total of 3496 oocytes. The day after follicle aspiration the oocytes were inspected for their pronuclei: 1418 oocytes were found to be fertilized $(38.4 \%)$ of which $97.0 \%$ developed into embryos. 1871 oocytes were unfertilized $(50.7 \%)$ of which $6.9 \%$ developed into embryos; 106 oocytes (2.9\%) had one pronucleus and $172(4.7 \%)$ were polypronucleated.

ET took place two or three days after follicle aspiration: 295 ETs with only two pronucleate embryos leading to 83 clinical pregnancies and 68 pregnancies with heartbeat (62 ongoing pregnancies beyond 13 weeks); 37 ETs with mixed embryos (embryos developed from fertilized and "unfertilized" oocytes) leading to 11 clinical pregnancies, all with heartbeat and all ongoing. Four of these contained more foetuses than the number of "fertilized" oocytes that were transferred and therefore must come from the apprently unfertilized oocytes. Seven additional ETs in which only embryos from "unfertilized" oocytes were transferred led to 2 ongoing pregnancies. In the remaining 16 ETs with transfer of at least one embryo from a 1 pronucleate zygote 5 clinical pregnancies occurred, 4 ongoing. So far 4 children from the 6 pregnancies of at least one embryo with 0 pronuclei have been born, all but one (anencephaly, diagnosed at 16 weeks) apparently healthy

The development of embryos from unfertilized oocytes can be a consequence of either parthenogenesis of early late fertilization (the pronuclei have already disappeared or not yet appeared). We conclude from our data that in case of absence of pronuclei, if the embryo has normal morphological characteristics there is a fair chance of the occurrence of a pregnancy, hence the embryo should be considered for transfer.

126. The Effects of Buserelin administration during hMG stimulation on oocyte maturity and embryo development

J. Smitz, M. Camus, N. Bollen, H. Tournaye, E. Van den Abbeel, C. Staesen, P. Devroey, and A. Van Steirteghem Centre for Reproductive Medicine, Academic Hospital - "Vrije Universiteit Brussel", Brussels, Belgium

Different investigators ${ }^{1}$ reported a lower embryo quality after GnRH agonist-hMG stimulation. This phenomenon could be abscribed to the high number of exogenous gonadotrophins (hMG) used, to a direct intra-Fallopian effect of the agonist or to the nature of the follicles that are required.

In this work we evaluated the effect of the agonist during the follicular stimulation with hMG. We randomized 49 women with tubal infertility who were stimulated for IVF. A first group of 29 patients (G1) was desensitised with a daily dose of $600 \mu \mathrm{g}$ Buserelin intranasally. When desensitisation was achieved the agonist was stopped and hMG stimulation was started. The second group $(n=20)$ of patients was desensitised with the same dose, but the agonist was continued during hMG stimulation until the ovulatory hCG dose. Both groups needed a similar amount of hMG ampoules during an equal number of days to achieve preovulatory follicles. No endogenous LH surges occurred in the G1 group, but the mean LH at the moment of hCG injection in this group $(3.7 \pm 0.6)$ was significantly higher $(\mathrm{p}<0.001)$ than in $\mathrm{G} 2(1.20 .1)$. The number of oocytes collected in G1 was $10.0( \pm 5.8)$ and in G2 $13.3( \pm 9.5)$ (NS). In G2 we observed a higher amount of slightly overmature oocytes in a higher number of patients $\left(\chi^{2}=17.9 ; \mathrm{p}<10^{-4}\right)$. Fertilization, cleavage and embryo scoring were not different in both groups. The pregnancy rate and the number of cryopreserved embryos was also not statistically different in both treatments. It is to early to evaluate the outcome of the frozen embryos. We conclude that in normo-ovulatory women where Buserelin was omitted during hMG stimulation after an initial desensitization, no endogenous $\mathrm{LH}$ rises occurred. Although a higher rate of overmature oocytes was recorded in the group where Buserelin was continued until ovulation, this phenomenon seemed to have no impact on subsequent embryo development and implantation.

$1_{\mathrm{J}}$. Testart et al. (1988) Contraception Fertilité Sexualité, 16, 669670; R. Brzyski et al (1988) Fertility Sterility 50, 917-921; E. Van den Abbeel et al. (1989) in Abstract book of the Annual ESHRE Meeting 1989, Malmő, Sweden, W3: 3p17. 
127. Timing of insemination according to ovulationtracking performed at home by the patient using LH slef tests

D. Weihs, M. Germond, A. Senn, H.J. Welti, and P. De Grandi Unité de Stérilité, Départment de Gynéco-Obstétrie, CHUV, Lausanne, Switzerland

After routine control cycle based on serum hormone analysis, normo-ovulating patients attending our unit for insemination with homologous (IAC) or donor (IAD) sperm were offered the possibility to perform detection of the luteinizing hormone $(\mathrm{LH})$ surge using a qualitative urinary test (OvuQUICK, Monoclonal Antibodies, U.S.A.). First and second morning urines were analyzed and recorded by the patient until the first positive result was found. The first insemination was then scheduled according to the disponibility of both the patient and the medical staff. A second or third insemination was eventually performed on subsequent days. Each cycle was reviewed retrospectively by one single physician of the unit who determined what should have been the optimal insemination date depending on whether the first, the second or both daily LH-tests were positive. These theoretical timings were then compared to the days of performed insemination(s).

Out of 151 initiated cycles, 38 had to be rejected for incomplete data recording or inconsistant appreciation by the patient. In 113 cycles (42 IAC, 21 patients, 3 pregnant; 71 IAD, 32 patients, 7 pregnant), 62 positive tests were obtained in the first urine (55\%) and 51 in the second (45\%). The following table presents the degree of coincidence per cycle between the theoretical (D) and the insemination (Di) days, as well as the distribution of the induced pregnancies (in parethesis).

$\begin{array}{clllll}n^{\circ} \text { of inseminations } & D i<D & D i=D & D i>D & \text { Di<D+Di>D } & \begin{array}{l}\text { Correct } \\ \text { timing (\%) }\end{array} \\ 1 & 20(0) & 15(2) & 1(0) & - & 42 \% a \\ 2-3 & 21(3)^{b} & 51(4)^{b} & 1(0) & 4(1) & 66 \% a \\ \text { a: } p \leq 0.05 ; ~ b: p>0.5 \text { (chi-square) } & & & \end{array}$

LH self test may be safely used to cover the ovulation period, provided 2 inseminations are performed. The pregnancy rates of this study (u) are comparable to those initiated after serum (s) monitoring of ovulation for IAC ( $u$ vs s: 7.1 vs $4.7 \%, p>0.7$ ) and IAD ( 9.7 vs $9.4 \%, \mathrm{p}>0.8)$ cycles.

\section{Hysteroscopic zygote intrafallopian transfer}

H. Kururmäki, K. Ratsula, A. Lähteermäki and O. Hovatta Infertility Clinic of the Finnish Population and Family Welfare Federation, Kalevankatu 16 D, SF-00100 Helsinki

The benefit of GIFT, ZIFT and TET to IVF is the later and more natural transfer of embryos to uterine cavity resulting in higher pregnancy rate. To avoid general anesthesia and laparoscopy, which earlier were needed for GIFT, ZIFT or TET procedures, Jansen and Anderson (Lancet 2: 309, 1987) have developed ultrasound guided transcervical technique for transfer of gametes, zygotes or embryos into the fallopian tubes. However, the operator cannot be sure what is the exact situation of the tip of the catheter when he injects the gametes, zygotes or embryos.
We have now tested a new hysterofibroscopic technique where the situation of the catheter tip is exactly known.

Nine patients with patent tubes were programmed to our normal IVF-stimulation (long buserelin-hMG scheme). HCG was given when the leading follicle reached $17 \mathrm{~mm}$ diameter, and ultrasound guided transvaginal follicle aspiration was done 34 hours later. During the next day hysteroscopy (Olympus HYP-P hysterofiberscope) was carried out using $\mathrm{CO}_{2}$ for uterine distention. Patients did not feel pain and they did not need any anesthesia. The tubal ostia were visualized and $2-4$ zygotes in pronuclear stage were loaded into the catheter (hysteroscopic insemination catheter sets, Cook, USA) and introduced through the hysteroscope's operating channel into the ostium of one tube. The pressure of $\mathrm{CO}_{2}$ was taken off and after 5 minutes stabilizing time, zygotes were injected into the fallopian tube. From nine patients, two have now ongoing pregnancies. These preliminary results show that this technique is usable, but a larger group study is needed to see the efficacy of this technique.

129. Treatment independent pregnancies among patients enrolled in a programmer of in vitro fertilization and embryo transfer

\section{S. Bonanomi, M. Germond, A. Senn, P. De Grandi}

Unité de Stérilité, Département de Gynécologie-Obstétrique, CHUV, 1011 Lausanne, Switzerland

Although the technique of in vitro fertilization and embryo transfer (IVF - ET) was developed initially for women with tubal occlusions, indication for such a treatment have been widened to other causes of infertility such as male factor, endometriosis or idiopathic infertility. As these indications are not absolute causes of sterility, one might expect treatment independent (TI) pregnancies to occur at higher rates among those patients than among the restricted tubal group.

The aim of this study was to review retrospectively the follow-up of the couples enrolled in our IVF-ET programme (Fertility- Sterility Unit, CHUV, Lausanne) between October 1985 and April 1990, and to determine the rates and circumstances of the TI pregnancies.

Among the 750 couples to which an IVF-ET attempt was proposed, $40 \mathrm{TI}$ pregnancies (5\%) were diagnosed either while the patients were on the waiting list or between two treatment cycles. These TI pregnancies were distributed among the following indications: 15 tubal $(37 \%), 9$ male $(23 \%), 6$ idiopathic (15\%), 10 multifactorial $(25 \%)$. These pregnancies were analyzed in respect to duration of infertility, age and time regarding the treatment and compared to those resulting from IVF-ET.

Our TI pregnancy rate $(5 \%)$ is low compared to the $8-16 \%$ published in the literature and indicates that widening the acceptance criteria for IVF-ET does not necessarily increase the TI pregnancy rate provided precise and rigorous diagnostic procedures are adopted before enrolling. This suggests that IVFET should - for economical and psychological reasons - be accessible only to those patients for which this therapy remains the only hope to conceive. 